\title{
A STUDY ON DIMENSIONAL ANALYSIS FOR AIR-WATER FLOW SYSTEM
}

\author{
Ashrafuzzaman Khan ${ }^{1}$ and Ashabul Hoque ${ }^{2}$ \\ ${ }^{1}$ Department of Applied Mathematics \\ University of Rajshahi, Rajshahi-6205, Bangladesh \\ E-mail: akhanmath@yahoo.com \\ and
${ }^{2}$ Department of Mathematics \\ University of Rajshahi, Rajshahi-6205, Bangladesh \\ E-mail: ashabulh@yahoo.com
}

Received 13.09.09 Accepted 19.06.10

\begin{abstract}
A simplified exposition and its consequence are given for the dimensional analysis. We have first discussed Buckingham's $\pi$ theorem, which provides a guide to the number of dimensions parameters required to specify any particular phenomenon. The influence of dimensional analysis on air-water flow system is described in details and is applied to scaling the measurements of void fraction distributions in vertical circular plunging jets. This study highlights the dimensional analysis on air-water flow system, which is a convenient technique for estimating the magnitudes of various terms in the governing equations.
\end{abstract}

Key words: Dimension analysis; Buckingham's theorem; Air-water flow; Void fraction.

\section{Introduction}

Dimensional analysis is a branch of mathematics, which deals with the dimensions of quantities. Each physical phenomenon can be explained by an equation giving the relationships between different quantities of dimensional or non-dimensional. In general, any variable present in a physical phenomenon will be a dimensional quantity (e.g. density, velocity, pressure etc.).

The dimensions of properties, which are involved in studying, related to hydraulics and fluid mechanics partial analysis is known as dimensional analysis. The studies of airwater flows are complex because of a large number of relevant equations is involved. To reduce the number of variables and also equations, dimensional analysis is necessary. Dimensional analysis alone, however, is inadequate, but the equations can serve as a supplement. The basis of dimensional is that, each quantity is made up of the fundamental dimensions of mass, length and time and that any relationship between variables must be homogeneous in these fundamental dimensions, the units attached to those quantities are called fundamental units.

In the study of air-water flows, the relevant parameters needed for any dimensional 
analysis come from the following groups: (a) fluid properties and physical constant, (b) flow geometry, (c) geometry of air supply system, (d) air-water flow properties, (e) upstream flow properties and (f) entrained air bubble characteristics.

Dimensional analysis is very much related to the air-water flow properties (Chanson et $a l^{4}{ }^{4}$ Liggett ${ }^{6}$, Thomson ${ }^{7}$ ). Although the dimensionless number was named after William Froude (1810-1879), several French researchers used it before $\left(\right.$ Chanson $\left.^{2}\right)$. Ferdinand Reech (1805-1880) introduced the dimensionless number for testing ships and propellers in 1852, and the number should really be called the Reech-Froude number. The mechanisms of air entrainment depend upon the jet velocity at impact, the physical properties of fluid, the jet nozzle design, the length of free-falling jet and the jet turbulence. For small velocities, air is entrained in the form of individual air bubbles and at larger jet velocities, large packets of air are entrained and broken up subsequently in the shear flow (e.g. Bin ${ }^{1}$, Chanson and Brattberg ${ }^{5}$ ).

It is the purpose of the present paper to attempt a simple exposition, setting forth the nature and uses of the dimensional analysis.

\section{Physical Properties of Air-Water Flow}

\subsection{Dimensional analysis}

Analytical and numerical studies of air entrapment and diffusion processes are complex because of the large number of relevant parameters and equations. The basic parameters needed for any dimensional analysis (Fig. 1) in hydraulic models are concerned in terms of a system having properties all of which can be grouped into three categories.

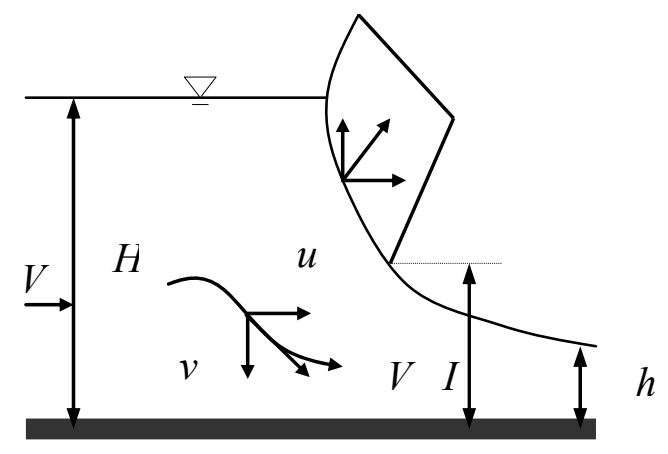

Figure 1: Sketch of basic flow parameters

Dimensional analysis method is based largely on the work of Fourier who developed the principle of dimensional homogeneity. Essentially this states that any equation is correct only if all terms of the equation have the same dimensions. More specifically, because the dimensions of any term can be represented as multiples of mass $(M)$, length $(L)$ and time $(T)$, it means that the exponents of the mass, length and time dimensions must be the same in each term of the equation. 


\subsection{Air-water flow}

In a nature free-surface aerated flows are encountered at waterfalls and when wave breaking occurs on the ocean surface. During the air entrainment, free surface becomes almost white by air bubbles, which is always visible to the naked eye (Fig. 2). The mechanisms of bubble entrainment depend upon the jet velocity at impact, the physical properties of fluid and jet turbulence $\left(\operatorname{Bin}^{1}\right)$. Many researchers showed interest to investigate of air entrainment in vertical plunging jets (hydraulic structure), but only a small number of researchers studied this in the surf zone.

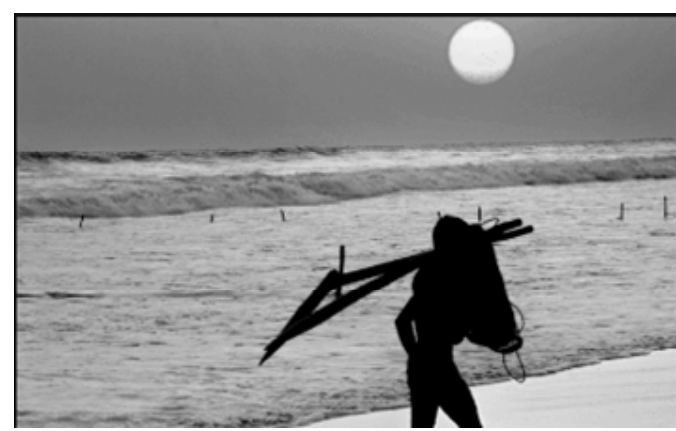

(a)

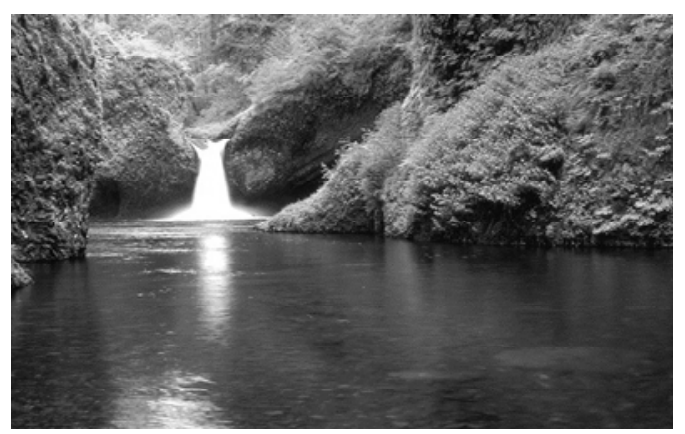

(b)

Figure 2: Photographs of air entrainment: (a) in the surf zone; (b) at a vertical plunging jet

Air-water flow properties consist of the void fraction distributions and velocity distributions. Chanson et al.4 presented the measurements of void fraction data and investigated the comparison among the three-scale model. Further, physical modeling of plunging jet flows remains subject to scaling effects which have not been properly explained.

\section{Application of Dimensional Analysis}

In practice, most hydraulic models are scaled with either a Froude or a Reynolds similitude, i.e. the selected dimensionless number is the same in the model and in the prototype. Buckingham's theorem provides a guide to the number of dimensions parameters required to specify any particular phenomenon. Taking into account all basic parameters, dimensional analysis yields;

$$
F_{1}\left(\rho, \mu, \sigma, E_{b}, g, L, V, \Delta P\right)=0
$$

There are eight basic parameters and the dimensions of these can be grouped into three categories. The Buckingham $\pi$-theorem implies that the quantities can be grouped into five $(5=8-3)$ independent dimensionless parameters:

$$
F_{2}\left(\frac{V}{\sqrt{g L}} ; \frac{\rho V^{2}}{\Delta P} ; \frac{\rho V L}{\mu} ; \frac{V}{\sqrt{\sigma / \rho L}} ; \frac{V}{\sqrt{E_{b} / \rho}}\right)=0
$$


That is, $\quad F_{2}(F r ; E u ; \operatorname{Re} ; W e ; M a)=0$

The first ratio is the Froude number Fr, characterizing the ration of the inertial force to gravity force. $\mathrm{Eu}$ is the Euler number, proportional to the ration of inertial force to pressure force. The third dimensionless parameter is the Reynolds number Re which characterizes the ratio of inertial force to viscous force. The Weber number $\mathrm{We}$ is proportional to the ratio of inertial force to capillary force. The last parameter is the Sarrau-Mach number, characterizing the ratio of inertial force to elastic force.

Any combination of the dimensionless numbers involved in Eq. (2) is also dimensionless and may be used to replace one of the combinations. It can be shown that one parameter can be replaced by the Morton number M0 $=(g \mu 4) /(\rho \sigma 3)$, also called liquid parameter, since:

$$
M_{0}=\frac{W e^{3}}{F r^{2} \operatorname{Re}^{4}}
$$

The Morton number is a function of fluid properties and gravity constant. For the same fluids (air and water) in both model and prototype, the Morton number is a constant.

Considering the simpler steady, the vertical circular plunging jet, Eq. (1) may be simplified into:

$$
F_{2}\left(C ; F r ; T u ; \frac{r}{d_{1}} ; \frac{x-x_{1}}{d_{1}} ; \frac{r}{d_{1}} ; \frac{x_{1}}{d_{1}} ; F r_{1} ; W e_{1} ; T u_{1} ; M_{0}\right)=0
$$

In Eq. (3), $C$ is the dimensionless parameter and subscript ' 1 ' is the inflow conditions. Equations (2) and (3) demonstrate the dynamic similarity of air bubble entrainment at plunging jets is impossible with geometrically similar models. In free-surface flow and wave motion, most laboratory studies are based upon a Froude similitude (Chanson2). But the entrapment of air bubbles and the mechanisms of air bubble breakup and coalescence are dominated by surface tension effects implying the need for Weber similitude. For geometrical similar models, Froude and Weber similarities can't be satisfied simultaneously.

Solving practical design problems in fluid mechanics usually requires both theoretical developments and experimental results. By grouping significant quantities into dimensionless parameters, it is possible to reduce the number of variables appearing and to make this compact result (equations or data plots) applicable to all similar situations.

\section{Example}

An example of the use of dimensional analysis and its advantages is given by considering the circular plunging jet, treated in the diffusion equation for this case:

The diffusion of air bubbles in a turbulent plunging jet flow can be approximated by an analytical solution of diffusion equation (Chanson 1997). For a circular plunging jet, the diffusion equation in cylindrical co-ordinates is: 


$$
\frac{\partial}{\partial x}\left(C * V_{x}\right)=\frac{1}{r} * \frac{\partial}{\partial r}\left(D_{r} * r * \frac{\partial C}{\partial r}\right)
$$

where $\mathrm{x}$ is the longitudinal distance measured from the nozzle, $\mathrm{r}$ is the normal direction, $\mathrm{C}$ is the void fraction, $\mathrm{Vx}$ is the longitudinal velocity component in the $\mathrm{x}$-direction and $\mathrm{Dr}$ is the turbulent diffusivity in the normal direction.

For a uniform velocity distribution, i.e. $\mathrm{Vx}=\mathrm{V} 0$ and diffusion co-efficient $\mathrm{Dr}$ independent of the longitudinal location then the simple diffusion equation is

$$
\frac{V_{0}}{D_{t}} * \frac{\partial}{\partial x} C=\frac{1}{r} * \frac{\partial}{\partial r}\left(r * \frac{\partial}{\partial r} C\right)
$$

where Dt is the diffusivity term averages the effects of the turbulent diffusion and of the longitudinal velocity gradient, i.e.

$$
D_{t}=D_{r} *\left(1+\frac{1}{C} *\left(\frac{\partial}{\partial x} C / \frac{\partial}{\partial x} V_{x}\right)\right)
$$

The boundary conditions are $\mathrm{C}(\mathrm{x}<0, \mathrm{r})=0$ and circular source of total strength Qa at $\left(x=0, r=r_{0}\right)$. Applying a superposition method, the solution of the air bubble diffusion equation becomes

$$
C=\frac{Q_{a}}{Q_{w}} * \frac{1}{4 * D^{\#} \frac{x-x_{1}}{r\left(C_{\max }\right)}} * \exp \left(-\frac{1}{4 * D^{\#}} * \frac{\left(\frac{r}{r\left(C_{\max }\right)}\right)^{2}+1}{\frac{x-x_{1}}{r\left(C_{\max }\right)}}\right) * I_{0}\left(\frac{1}{2 * D^{\#}} * \frac{\frac{r}{r\left(C_{\max }\right)}}{\frac{x-x_{1}}{r\left(C_{\max }\right)}}\right)
$$

where $\mathrm{x}$ is the longitudinal distance measured from the nozzle, $\mathrm{x} 1$ is water jet length, $\mathrm{r}$ is the normal direction, $\mathrm{r} 1$ is the impact water jet thickness, Qw is the flow rate, Qa is the volume of air flux, D\# is a dimensionless diffusivity (Dt/(V1r1)) and I0 is the modified Bessel function of the first kind of order zero (Chanson3).

Figures 3 and 4 represent the typical dimensionless void fraction data and dimensional bubble count rates for vertical circular plunging jet, respectively. The data of void fraction of Chanson et al.4 may be fitted by a simple analytical solution of the advective diffusion equation (7) for air bubbles. 


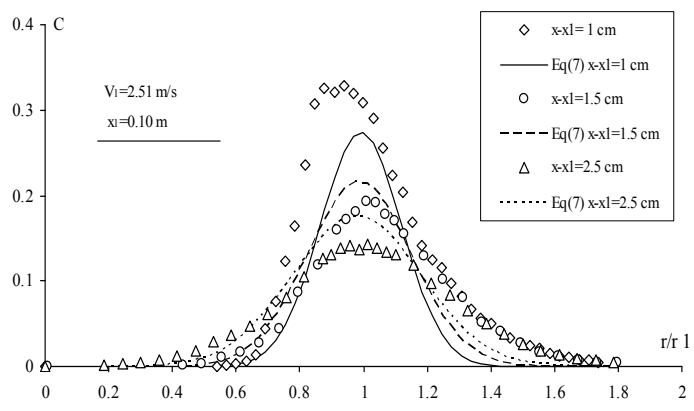

$$
\mathrm{V}_{1}=2.51 \mathrm{~m} / \mathrm{s}
$$

Figure 3: Distributions of void fraction $C$ for vertical plunging jet
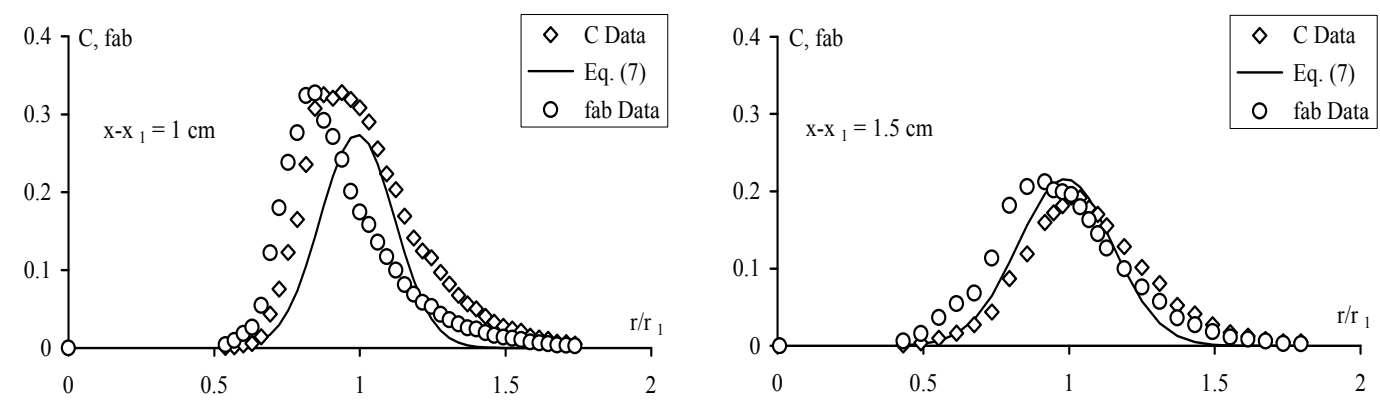

$$
\mathrm{x}-\mathrm{x}_{1}=1 \mathrm{~cm}
$$

$$
\mathrm{x}-\mathrm{X}_{1}=1.5 \mathrm{~cm}
$$

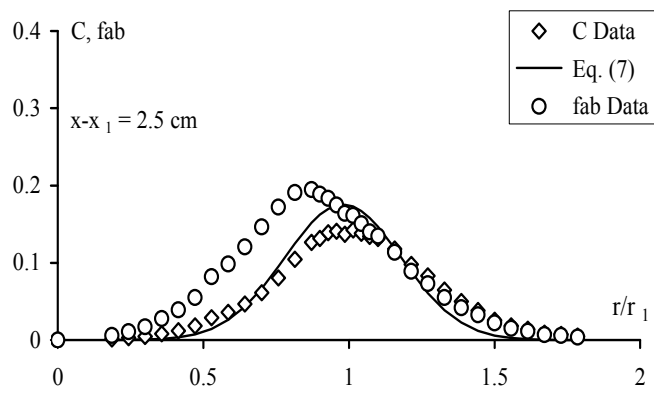

$$
\mathrm{x}-\mathrm{x}_{1}=2.5 \mathrm{~cm}
$$

Figure 4: Distributions of dimensionless bubble count rate $f a b=F r_{l} / V_{l}$

\section{Summary and Conclusion}

Dimensional analysis for air water flow has been presented systematically based upon a Buckingham's $\pi$-theorem. The dimensionless equations of fluid mechanics are particularly important since their solution is independent of the units of measurement and 
the scale of the problem. The basis of dimensional is that each quantity is made up of the fundamental dimensions of mass, length and time and that any relationship between variables must be homogeneous in these fundamental dimensions. Two important proposals are given; (i) the dimensionless equations highlight the important effects in any flow problem and indicate which terms are small enough to be neglected if the complete equations cannot be solved, (ii) similarity and dissimilarity between two related flow systems, model and prototype, are indicated by the dimensionless equations. In practice, most hydraulic models are scaled with either a Froude or a Reynolds similitude: i.e. the selected dimensionless number is the same in the model and in prototype (i.e. full-scale).

The equations are an important guide for experiment and experimentation is essential in fluid mechanics. The equations indicate the correct approximation; the magnitude of the error, and sometimes the verification of the approximation must come from experiment. This study demonstrated the dimensional analysis on air-water flow system which is a convenient technique for estimating the magnitudes of various terms in the governing equations.

\section{Notation}

The following symbols are used in this paper:

C air concentration defined as the volume of air per unit volume of air and water; it is also called void fraction;

$C_{\max } \quad$ maximum void fraction in a cross-section;

$D_{r} \quad$ turbulent diffusivity in the normal direction;

$D_{t} \quad$ turbulent diffusivity of air bubbles in air-water flow;

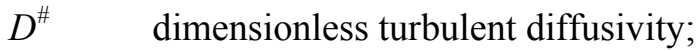

$d_{l} \quad$ jet thickness at plunge point;

$E_{b} \quad$ pipe roughness;

$\mathrm{Eu} \quad$ Euler number;

$F_{r} \quad$ Froude number;

$g \quad$ gravity constant;

$I_{0} \quad$ modified Bessel function of the first kind of order zero;

L longitudinal distance;

Ma Mach number;

$M_{0} \quad$ Morton number;

$P \quad$ pressure;

$Q_{a} \quad$ volume of air flux; 
$Q_{w} \quad$ flow rate;

$r \quad$ radial distance from the centerline;

$r_{1} \quad$ impact water jet thickness;

Re Reynolds number;

$\mathrm{Tu} \quad$ turbulence intensity;

$T u_{1} \quad$ turbulence intensity at jet impact measured on the centreline;

V velocity;

$V_{x} \quad$ longitudinal velocity component in the $x$-direction;

We Weber number;

$W e_{1} \quad$ Weber number at impingement;

$x \quad$ longitudinal distance measured from the nozzle;

$x_{1} \quad$ distance between nozzle and impingement point;

Greek symbols

$\mu \quad$ dynamic viscosity;

$\rho$ density;

$\sigma \quad$ surface tension between air and water;

Abbreviations

$\partial / \partial r \quad$ partial derivative with respect to $r$;

$\partial / \partial x \quad$ partial derivative with respect to $x$;

\section{REFERENCES}

1. Bin, A. K., Gas entrainment by plunging liquid jets, Chem. Eng. Science, 48(21) (1993) 3585-3630.

2. Chanson, H., The hydraulics of open channel flows: an introduction, Butterworth-Heinemann, Oxford, UK, (1999) $512 \mathrm{pp}$.

3. Chanson, H., Air bubble entrainment in free-surface turbulent shear flows, Academic Press, London, UK, (1997) 401 pp.

4. Chanson, H., Aoki, S., and Hoque, A., Physical modeling and similitude of air bubble entrainment at vertical circular plunging jets, Chemical Eng. Sci., 59 (2004) 747-758.

5. Chanson, H., and Brattberg T., Air entrainment by two-dimensional plunging jets: the impingement region and the very-near flow field, Proc. Of ASME Fluids Eng. Conf., USA (1998) 8 pp.

6. Liggett, J. A., Fluid mechanics, McGraw-Hill, (1994) 495 pp.

7. Milne-Thomson, L. M., Theoretical Hydrodynamics, The Macmillan Press, London, (1968) 741 pp. 